\title{
Coefficient Estimates for Starlike and Convex Classes of m-fold Symmetric Bi-univalent Functions
}

\author{
S. Sivasubramanian ${ }^{1}$, P.Gurusamy ${ }^{2, *}$ \\ ${ }^{1}$ Department of Mathematics, University College of Engineering Tindivanam, Anna University, Tindivanam, India \\ ${ }^{2}$ Department of Mathematics, Velammal Engineering College, Surapet, Chennai, India \\ *Corresponding author: gurusamy65@gmail.com
}

Received March 31, 2015; Revised May 31, 2015; Accepted August 02, 2015

\begin{abstract}
In an article of Pommerenke [10] he remarked that, for an $m$-fold symmetric functions in the class $\mathcal{P}$, the well known lemma stated by Caratheodary for a one fold symmetric functions in $\mathcal{P}$ still holds good. Exploiting this concept, we introduce certain new subclasses of the bi-univalent function class $\sigma$ in which both $f$ and $f^{-1}$ are $m$-fold symmetric analytic with their derivatives in the class $\mathcal{P}$ of analytic functions. Furthermore, for functions in each of the subclasses introduced in this paper, we obtain the coefficient bounds for $\left|a_{m+1}\right|$ and $\left|a_{2 m+1}\right|$. We remark here that the concept of $m$-fold symmetric bi-univalent is not in the literature and the authors hope it will make the researchers interested in these type of investigations in the forseeable future. By the working procedure and the difficulty involved in these procedures, one can clearly conclude that there lies an unpredictability in finding the coefficients of a $m$-fold symmetric bi-univalent functions.
\end{abstract}

Keywords: analytic functions, univalent functions, bi-univalent functions, $m$-fold symmetric functions, subordination

Cite This Article: S. Sivasubramanian, and P.Gurusamy, "Coefficient Estimates for Starlike and Convex Classes of $\boldsymbol{m}$-fold Symmetric Bi-univalent Functions." Turkish Journal of Analysis and Number Theory, vol. 3, no. 3 (2015): 83-86. doi: 10.12691/tjant-3-3-3.

\section{Introduction}

Let $\mathcal{A}$ denote the class of functions of the form:

$$
\left.f(z)=z+\sum_{n=2}^{\infty}\right\rceil_{n} z^{n}, \quad z \in \mathbb{U},
$$

which are analytic in the open unit disk $\mathbb{U}=\{z \in \mathbb{C}:|z|<$ $1\}$. Further, by $\mathcal{S}$, we mean the class of all functions in $\mathcal{A}$ which are univalent in $\mathbb{U}$. For more details on univalent functions, see [3]. It is well known that every function $f \in \mathcal{S}$ has an inverse $f^{-1}$, defined by

$$
f^{-1}(f(z))=z \quad(z \in \mathbb{U})
$$

and

$$
f\left(f^{-1}(w)\right)=w \quad\left(|w|<r_{0}(f) ; r_{0}(f) \geq \frac{1}{4}\right) .
$$

Indeed, the inverse function may have an analytic continuation to $\mathbb{U}$, with

$$
\begin{aligned}
f^{-1}(w)= & w-a_{2} w^{2}+\left(2 a_{2}^{2}-a_{3}\right) w^{3} \\
& -\left(5 a_{2}^{3}-5 a_{2} a_{3}+a_{4}\right) w^{4}+\cdots .
\end{aligned}
$$

A function $f \in \mathcal{A}$ is said to be bi-univalent in $\mathbb{U}$ if both $f$ and $f^{-1}$ are univalent in $\mathbb{U}$. Let $\sigma$ denote the class of $b i$ univalent functions in $\mathbb{U}$, given by equation (1). An analytic function $f$ is subordinate to an analytic function $g$, written $f(z) \prec g(z)$, provided there is an analytic function $w$ defined on $\mathbb{U}$ with $w(0)=0$ and $|w(z)|<1$ satisfying $f(z)=g(w(z))$. Lewin [8] investigated the class of bi-univalent functions $\sigma$ and obtained a bound $\left|a_{2}\right| \leq 1.51$. Motivated by the work of Lewin [8], Brannan and Clunie [1] conjectured that $\left|a_{2}\right| \leq \sqrt{2}$. Some examples of bi-univalent functions are $\frac{z}{1-z}, \frac{1}{2} \log \left(\frac{1+z}{1-z}\right)$ and $-\log (1-z)$ (see also the work of Srivastava et al. [11]). The coefficient estimate problem for each of the following Taylor-Maclaurin coefficients: $\left|a_{n}\right| \quad(n \in \mathbb{N}, n \geq 3)$ is still open([11]). In recent times, the study of bi-univalent functions gained momentum mainly due to the work of Srivastava et al. [11]. Motivated by this, many researchers (see $[4,11,12,13,14,15,17])$ recently investigated several interesting subclasses of the class $\sigma$ and found non-sharp estimates on the first two Taylor-Maclaurin coefficients. For each function $f$ in $\mathcal{S}$, the function $h(z)=\sqrt[m]{f\left(z^{m}\right)}$ is univalent and maps the unit disk $\mathbb{U}$ into a region with $m$ fold symmetry. A function is $m$-fold symmetric (see [10]) if it has the normalized form

$$
f(z)=z+\sum_{k=1}^{\infty} \uparrow_{m k+1} z^{m k+1}, \quad z \in \mathbb{U},
$$

and we denote the class of $m$-fold symmetric univalent functions by $\mathcal{S}_{m}$, which are normalized by the above series expansion. In fact, the functions in the class $\mathcal{S}$ are one fold symmetric. Analogous to the concept of $m$-fold symmetric univalent functions, one can think of the concept of $m$ fold symmetric bi-univalent functions in a natural way. Each function in the class $f$ in $\sigma$, generates an $m$-fold symmetric bi-univalent function for each integer $m$. The normalized form of $f$ is given as in (5) and $f^{-1}$ is given as follows. 


$$
\begin{aligned}
& g(w)=w-a_{m+1} w^{m+1}+\left[(m+1) a_{m+1}^{2}-a_{2 m+1}\right] w^{2 m+1} \\
& -\left[\begin{array}{l}
\frac{1}{2}(m+1)(3 m+2) a_{m+1}^{3} \\
-(3 m+2) a_{m+1} a_{2 m+1}+a_{3 m+1}
\end{array}\right] w^{3 m+1}+\cdots
\end{aligned}
$$

where $f^{-1}=g$. We denote the class of $m$-fold symmetric bi-univalent functions by $\sigma_{m}$. For $m=1$, the formula (6) coincides with the formula (4) of the class $\sigma$. Denote also, by $\mathcal{P}$, the class of analytic functions of the form $p(z)=$ $1+p_{1} z+p_{2} z^{2}+\cdots$ such that $\operatorname{Re}(p(z))>0$ in $\mathbb{U}$. In view of Pommerenke [10], the $m$-fold symmetric function in the class $\mathcal{P}$ is of the form

$$
p(z)=1+c_{m} z^{m}+c_{2 m} z^{2 m}+c_{3 m} z^{3 m}+\cdots .
$$

It is assumed that $\varphi$ is an analytic functions with positive real part in the unit disk $\mathbb{U}$, with $\varphi(0)=$ $1, \varphi^{\prime}(0)>0$ and $\varphi(\mathbb{U})$ is symmetric with respect to the real axis. Such a function has a series expansion of the form

$$
\varphi(z)=1+B_{1} z+B_{2} z^{2}+B_{3} z^{3}+\ldots\left(B_{1}>0\right)
$$

Suppose that $u(z)$ and $v(z)$ are analytic functions in the unit disk $\mathbb{U}$ with $u(0)$ and $v(0)=0,|u(z)|<$ $1,|v(z)|<1$ and suppose that

$$
u(z)=b_{m} z^{m}+b_{2 m} z^{2 m}+b_{3 m} z^{3 m}+\cdots
$$

and

$$
v(w)=c_{m} w^{m}+c_{2 m} w^{2 m}+c_{3 m} w^{3 m}+\cdots .
$$

We observe that

$$
\left|b_{m}\right| \leq 1,\left|b_{2 m}\right| \leq 1-\left|b_{m}\right|^{2},\left|c_{m}\right| \leq 1 \text { and }\left|c_{2 m}\right| \leq 1-\left|c_{m}\right|^{2} .
$$

By a simple computations, we have

$$
\varphi(u(z))=1+B_{1} b_{m} z^{m}+\left(B_{1} b_{2 m}+B_{2} b_{m}^{2}\right) z^{2 m}+\cdots(|z|<1)
$$

and

$$
\varphi(v(w))=1+B_{1} c_{m} w^{m}+\left(B_{1} c_{2 m}+B_{2} c_{m}^{2}\right) w^{2 m}+\cdots(|w|<1) .
$$

Motivated essentially by the work of Ma and Minda [9], we introduce some new subclasses of $m$-fold symmetric bi-univalent functions and obtain coefficient bounds of $\left|a_{m+1}\right|$ and $\left|a_{2 m+1}\right|$ for functions in these classes. The results presented in this paper improve the earlier results of Frasin and Aouf [4], Srivastava et al. [11] for the case of one fold symmetric functions.

\section{Coefficient Estimates for the Function Class $S T_{\sigma, m}(\varphi)$}

Definition 2.1 A function $f(z)$, given by (5), is said to be in the class $S T_{\sigma, m}(\varphi)$, if the following conditions are satisfied:

$$
\begin{aligned}
& f \in \sigma_{m}, \frac{z f^{\prime}(z)}{f(z)} \prec \varphi(z) \\
& \text { and } \frac{w g^{\prime}(w)}{g(w)} \prec \varphi(w), g(w)=f^{-1}(w)
\end{aligned}
$$

For the special choices of the function $\varphi(z)$ and for the choice of $m=1$, our class reduces to the following.

1. For $m=1$ and $\varphi(z)=\left(\frac{1+z}{1-z}\right)^{\gamma}, \quad(0<\gamma \leq 1)$, $S T_{\sigma, 1}\left(1,\left(\frac{1+z}{1-z}\right)^{\gamma}\right) \equiv S T_{\sigma}(\gamma),(0<\gamma \leq 1)$, the class of strongly bi-starlike functions of order $\gamma$ studied by Brannan and Taha [2].

2. For $m=1$ and $\varphi(z)=\frac{1+(1-2 \alpha) z}{1-z}(0 \leq \gamma<1)$, $S T_{\sigma, 1}\left(1,\left(\frac{1+(1-2 \gamma) z}{1-z}\right)\right) \equiv S_{\sigma}^{*}(\gamma),(0 \leq \gamma<1)$, the class of bi-starlike functions of order $\alpha$ studied by Brannan and Taha [2].

We first state and prove the following theorem.

Theorem 2.1 Let $f(z)$, given by (5), be in the class $S T_{\sigma, m}(\varphi)$.

Then

$$
\left|a_{m+1}\right| \leq \frac{B_{1} \sqrt{B_{1}}}{m \sqrt{B_{1}+\left|B_{1}^{2}-B_{2}\right|}}
$$

and

$$
\left|a_{2 m+1}\right| \leq \begin{cases}\frac{(1+m) B_{1}}{2 m^{2}} & \text { if }\left|B_{2}\right| \leq B_{1} \\ \frac{(1+m)\left(\left|B_{1}^{2}-B_{2}\right| B_{1}+\left|B_{2}\right| B_{1}\right)}{2 m^{2}\left(\left|B_{1}^{2}-B_{2}\right|+B_{1}\right)} & \text { if }\left|B_{2}\right|>B_{1}\end{cases}
$$

Proof. Let $f \in S T_{\sigma, m}(\varphi)$ and $g=f^{-1}$. Then there are analytic functions $u, v: \mathbb{U} \rightarrow \mathbb{U}$, with $u(0)=v(0)=0$ satisfying

$$
\frac{z f^{\prime}(z)}{f(z)}=\varphi(u(z)) \text { and } \frac{w g^{\prime}(w)}{g(w)}=\varphi(v(w)) .
$$

Since

$\frac{z f^{\prime}(z)}{f(z)}=1+m a_{m+1} z^{m}+\left(2 m a_{2 m+1}-m a_{m+1}^{2}\right) z^{2 m}+\cdots$

and

$$
\frac{w g^{\prime}(w)}{g(w)}=1-m a_{m+1} w^{m}+\left(\begin{array}{l}
m(2 m+1) a_{m+1}^{2} \\
-2 m a_{2 m+1}
\end{array}\right) w^{2 m}+\cdots,
$$

it follows from (12), (13) and (16) that

$$
\begin{gathered}
m a_{m+1}=B_{1} b_{m} \\
2 m a_{2 m+1}-m a_{m+1}^{2}=B_{1} b_{2 m}+B_{2} b_{m}^{2}, \\
-m a_{m+1}=B_{1} c_{m}
\end{gathered}
$$

and

$$
m(2 m+1) a_{m+1}^{2}-2 m a_{2 m+1}=B_{1} c_{2 m}+B_{2} c_{m}^{2} .
$$

From (17) and (19), we get

$$
c_{m}=-b_{m} \text {. }
$$

By adding (18) and (20) and in view of the computations using (17) and (21), we get

$$
2 m^{2}\left(B_{1}^{2}-B_{2}\right) a_{m+1}^{2}=B_{1}^{3}\left(b_{2 m}+c_{2 m}\right) .
$$

Further, (21), (22), together with (11), gives

$$
\left|m^{2}\left(B_{1}^{2}-B_{2}\right) a_{m+1}^{2}\right| \leq B_{1}^{3}\left(1-\left|b_{m}\right|^{2}\right) .
$$

Now from (17) and (23), we get

where the function $g$ is defined by (6). 


$$
\left|a_{m+1}\right| \leq \frac{B_{1} \sqrt{B_{1}}}{m \sqrt{B_{1}+\left|B_{1}^{2}-B_{2}\right|}}
$$

as asserted in (14).

By simple calculations from (18) and (20) using with the equations (17) and (21), we get

$$
4 m^{2} a_{2 m+1}=(1+2 m) B_{1} b_{2 m}+B_{1} c_{2 m}+2(1+m) B_{2} b_{m}^{2}
$$

Then using the equation (11) in (24), we get

$$
\left|a_{2 m+1}\right| \leq \frac{(1+m) B_{1}}{2 m^{2}}+\frac{(1+m)\left(\left|B_{2}\right|-B_{1}\right)\left|b_{m}\right|^{2}}{2 m^{2}} .
$$

Since

$$
\left|b_{m}\right|^{2} \leq \frac{B_{1}}{\left|B_{1}^{2}-B_{2}\right|+B_{1}}
$$

substituting (26) in (25), we get

$$
\left|a_{2 m+1}\right| \leq \begin{cases}\frac{(1+m) B_{1}}{2 m^{2}} & \text { if }\left|B_{2}\right| \leq B_{1} \\ \frac{(1+m)\left(\left|B_{1}^{2}-B_{2}\right| B_{1}+\left|B_{2}\right| B_{1}\right)}{2 m^{2}\left(\left|B_{1}^{2}-B_{2}\right|+B_{1}\right)} & \text { if }\left|B_{2}\right|>B_{1}\end{cases}
$$

as asserted in (15). This completes the proof of Theorem 2.1 .

For the case of one fold symmetric functions, Theorem 2.1 reduces to the coefficient estimates for Ma-Minda bistarlike functions in Srivastava et al [11].

Corollary 2.1 Let $f(z)$, given by (5), be in the class $S T_{\sigma}(\varphi)$. Then

$$
\left|a_{2}\right| \leq \frac{B_{1} \sqrt{B_{1}}}{\sqrt{\left|B_{1}^{2}+\left(B_{1}-B_{2}\right)\right|}}
$$

and

$$
\left|a_{3}\right| \leq B_{1}+\left|B_{2}-B_{1}\right| .
$$

For the case of one fold symmetric functions and for the class of strongly starlike functions, the function $\varphi$ is given by

$$
\varphi(z)=\left(\frac{1+z}{1-z}\right)^{\gamma}=1+2 \gamma z+2 \gamma^{2} z^{2}+\cdots,(0<\gamma \leq 1)
$$

which gives $B_{1}=2 \gamma$ and $B_{2}=2 \gamma^{2}$. Hence Theorem 2.1 reduce to the result in Brannan and Taha [2].

Corollary 2.2 [2] Let $f(z)$, given by (5), be in the class $S T_{\sigma}(\varphi)$. Then

$$
\left|a_{2}\right| \leq \frac{2 \gamma}{\sqrt{1+\gamma}}
$$

and

$$
\left|a_{3}\right| \leq 2 \gamma^{2} \text {. }
$$

For the case of one fold symmetric functions and for the class of strongly starlike functions, the function $\varphi$ is given by

$$
\varphi(z)=1+2(1-\gamma) z+2(1-\gamma)^{2} z^{2}+\cdots
$$

then $B_{1}=B_{2}=2(1-\gamma)$, and the Theorem 2.1 reduce to the result in Brannan and Taha [2].

Corollary 2.3 [2] Let $f(z)$, given by (5), be in the class $S T_{\sigma}(\varphi)$. Then

$$
\left|a_{2}\right| \leq \sqrt{2(1-\gamma)}
$$

and

$$
\left|a_{3}\right| \leq \sqrt{2(1-\gamma)}
$$

\section{Coefficient Bound for the Function Class $\mathcal{C} \mathcal{V}_{\sigma, m}(\lambda, \varphi)$}

Definition 3.1 A function $f(z)$, given by (5), is said to be in the class $\mathcal{C} \mathcal{V}_{\sigma, m}(\lambda, \varphi)$, if the following conditions are satisfied:

$$
f \in \sigma_{m}, 1+\frac{z f^{\prime \prime}(z)}{f^{\prime}(z)} \prec \varphi(z)
$$

and

$$
1+\frac{w g^{\prime \prime}(w)}{g^{\prime}(w)} \prec \varphi(w),
$$

where the function $g$ is defined by (6).

For one fold symmetric, a function in the class $\mathcal{C} \mathcal{V}_{\sigma, m}(\lambda, \varphi)$ is called bi-Mocanu-convex function of MaMinda type. For the special choices of the function $\varphi(z)$, and for the choice of $m=1$, our class reduces to the following.

1. For $m=1$ and $\varphi(z)=\frac{1+(1-2 \gamma) z}{1-z},(0 \leq \gamma<1)$, $C V_{\sigma, 1}\left(1,\left(\frac{1+z}{1-z}\right)^{\gamma}\right) \equiv C V_{\sigma}(\gamma)$, the class of strongly biconvex functions of order $\gamma$ studied by Brannan and Taha [2].

2. For $m=1$ and $\varphi(z)=\frac{1+(1-2 \gamma) z}{1-z}(0 \leq \gamma<1)$, $C V_{\sigma, 1}\left(1,\left(\frac{1+(1-2 \gamma) z}{1-z}\right)\right) \equiv C_{\sigma}(\gamma),(0 \leq \gamma<1)$, the class of bi-convex functions of order $\gamma$ studied by Brannan and Taha [2].

Theorem 3.1 Let $f(z)$, given by (5), be in the class $\mathcal{C} \mathcal{V}_{\sigma, m}(\lambda, \varphi), \lambda \geq 0$. Then

$$
\left|a_{m+1}\right| \leq \frac{B_{1} \sqrt{B_{1}}}{m \sqrt{(1+m)^{2} B_{1}+\left|(1+m) B_{1}^{2}-(1+m)^{2} B_{2}\right|}},
$$

and

$$
\left|a_{2 m+1}\right| \leq \begin{cases}\frac{B_{1}}{2 m^{2}} & \text { if }\left|B_{2}\right| \leq B_{1} \\
{\left[\begin{array}{l}
\left.\left|B_{1}^{2}-(1+m) B_{2}\right| B_{1}\right] \\
+(1+m)\left|B_{2}\right| B_{1}
\end{array}\right]} & \text { if }\left|B_{2}\right|>B_{1} \\
2 m^{2}\left(\begin{array}{l}
\left.\left|B_{1}^{2}-(1+m) B_{2}\right|\right) \\
+(1+m) B_{1}
\end{array}\right) & \end{cases}
$$

Proof. Let $f \in \mathcal{C} \mathcal{V}_{\sigma, m}(\lambda, \varphi)$. Then there are analytic functions $u, v: \mathbb{U} \rightarrow \mathbb{U}$, with $u(0)=v(0)=0$ satisfying

$1+\frac{z f^{\prime \prime}(z)}{f^{\prime}(z)}=\varphi(u(z))$ and $1+\frac{w g^{\prime \prime}(w)}{g^{\prime}(w)}=\varphi(v(w))$.

Since

$$
\begin{aligned}
& 1+\frac{z f^{\prime \prime}(z)}{f^{\prime}(z)}=1+m(1+m) a_{m+1} z^{m} \\
& +\left(2 m(1+2 m) a_{2 m+1}-m(1+m)^{2} a_{m+1}^{2}\right) z^{2 m}+\cdots
\end{aligned}
$$

and 


$$
\begin{aligned}
& 1+\frac{w g^{\prime \prime}(w)}{g^{\prime}(w)}=1-m(1+m) a_{m+1} w^{m} \\
& +\left(\begin{array}{l}
m(1+m)(3 m+1) a_{m+1}^{2} \\
-2 m(1+2 m) a_{2 m+1}
\end{array}\right) w^{2 m}+\cdots
\end{aligned}
$$

from (12), (13), and (36), we get

$$
\begin{gathered}
m(1+m) a_{m+1}=B_{1} b_{m}, \\
2 m(1+2 m) a_{2 m+1}-m(1+m)^{2} a_{m+1}^{2}=B_{1} b_{2 m}+B_{2} b_{m}^{2}, \\
-m(1+m) a_{m+1}=B_{1} c_{m},
\end{gathered}
$$

and

$$
\begin{aligned}
& m(1+m)(3 m+1) a_{m+1}^{2}-2 m(1+2 m) a_{2 m+1} \\
& =B_{1} c_{2 m}+B_{2} c_{m}^{2} .
\end{aligned}
$$

From (37) and (39), we get

$$
c_{m}=-b_{m} \text {. }
$$

By adding the equations (38) and (40), in view of computations using (37) and (41), we get

$$
\begin{aligned}
& 2 m^{2}(1+m)\left(B_{1}^{2}-(1+m) B_{2}\right) a_{m+1}^{2} \\
& =B_{1}^{3}\left(b_{2 m}+c_{2 m}\right) .
\end{aligned}
$$

Further, from the equations (41), (42), together with (11), we have

$$
\left|(1+m) m^{2}\left(B_{1}^{2}-(1+m) B_{2}\right) a_{m+1}^{2}\right| \leq B_{1}^{3}\left(1-\left|b_{m}\right|^{2}\right) .
$$

Now from (37) and (43), we get

$$
\left|a_{m+1}\right| \leq \frac{B_{1} \sqrt{B_{1}}}{m \sqrt{(1+m)^{2} B_{1}+\left|(1+m) B_{1}^{2}-(1+m)^{2} B_{2}\right|}}
$$

as asserted in(34). By simple calculations from (38) and (40) using with the equations (37) and (41), we get

$$
\begin{aligned}
& 4 m^{2}(1+2 m) a_{2 m+1} \\
& =(1+3 m) B_{1} b_{2 m}+(1+m) B_{1} c_{2 m}+2(1+2 m) B_{2} b_{m}^{2} .
\end{aligned}
$$

Then using the equation (11) in (44), we get

$$
\left|a_{2 m+1}\right| \leq \frac{B_{1}}{2 m^{2}}+\frac{\left(\left|B_{2}\right|-B_{1}\right)\left|b_{m}\right|^{2}}{2 m^{2}} \text {. }
$$

Since

$$
\left|b_{m}\right|^{2} \leq \frac{(1+m) B_{1}}{\left|B_{1}^{2}-(1+m) B_{2}\right|+(1+m) B_{1}},
$$

substituting (46) in (45), we get

$$
\left|a_{2 m+1}\right| \leq \begin{cases}\frac{B_{1}}{2 m^{2}} & \text { if }\left|B_{2}\right| \leq B_{1} \\ \frac{B_{1}^{2}-(1+m) B_{2}\left|B_{1}+(1+m)\right| B_{2} \mid B_{1}}{2 m^{2}\left(\left|B_{1}^{2}-(1+m) B_{2}\right|+(1+m) B_{1}\right)} & \text { if }\left|B_{2}\right|>B_{1}\end{cases}
$$

as asserted in (35).

For one fold symmetric functions then, Theorem 3.1 gives the coefficient for Ma-Minda bi-convex functions in Brannan and Taha [2]
Corollary 3.1 [2] Let $f(z)$, given by (5), be in the class $C T_{\sigma}(\varphi)$. Then

$$
\left|a_{2}\right| \leq \frac{B_{1} \sqrt{B_{1}}}{\sqrt{\left|2 B_{1}^{2}+4\left(B_{1}-B_{2}\right)\right|}}
$$

and

$$
\left|a_{3}\right| \leq \frac{B_{1}+\left|B_{2}-B_{1}\right|}{2} .
$$

For the case of one fold symmetric functions and for the class of strongly starlike functions, the function $\varphi$ is given by

$$
\varphi(z)=1+2(1-\gamma) z+2(1-\gamma)^{2} z^{2}+\cdots .
$$

then $B_{1}=B_{2}=2(1-\gamma)$, and the Theorem 3.1 reduce to the result in Brannan and Taha[2].

Corollary 3.2 [2] Let $f(z)$, given by (5), be in the class $\mathrm{CT}_{\sigma}(\varphi)$. Then

$$
\left|a_{2}\right| \leq 2(1-\gamma)
$$

and

$$
\left|a_{3}\right| \leq 1-\gamma
$$

\section{References}

[1] D. A. Brannan and J. G. Clunie, Aspects of contemporary complex analysis, Academic Press, London, 1980.

[2] D. A. Brannan and T. S. Taha, On some classes of bi-univalent functions, Stud.Univ.Babes-Bolyai Math. 31(1986), 70-77.

[3] P. L. Duren, Univalent functions, Springer-Verlag, New York, Berlin, Hiedelberg and Tokyo, 1983.

[4] B. A. Frasin and M. K. Aouf, New subclasses of bi-univalent functions, Appl. Math. Lett. 24 (2011), 1569-1573.

[5] J. Sokol, On a condition for $\alpha$ - starlikeness, J. Math. Anal. Appl. 352(2009), 696-701.

[6] M . K . Aouf, J . Sokol and J . Dziok, On a subclass of strongly starlike functions, Appl. Math. Lett. 24 (2011), 27-32.

[7] J . Sokol, A certain class of starlike functions, Comput. Math. Appl, 62(2011), 611-619.

[8] M. Lewin, On a coefficient problem for bi-univalent functions, Proc. Amer. Math. Soc. 18 (1967), 63-68.

[9] W. C. Ma, and D. Minda, A unified treatment of some special classes of unvalent functions, in Proceedings of the Conference on complex analysis. Tianjin (1992), 157-169.

[10] Ch.Pommerenke, On the coefficients of close-to-convex functions, Michigan. Math. J. 9 (1962), 259-269.

[11] H. M. Srivastava, A. K. Mishra and P. Gochhayat, Certain subclasses of analytic and bi-univalent functions, Appl. Math. Lett. 23 (2010), 1188-1192.

[12] H. M. Srivastava, S. Bulut, M. Cagler and N. Yagmur, Coefficient estimates for a general subclass of analytic and bi-univalent functions, Filomat. 27 (2013), 831-842.

[13] Q.-H. Xu, H. M. Srivastava and Z. Li, A certain subclass of analytic and close-to-convex functions, Appl. Math. Lett. 24 (2011), 396-â€"401.

[14] Q.-H. Xu, Y.-C. Gui and H. M. Srivastava, Coefficient estimates for a certain subclass of analytic and bi-univalent functions, Appl. Math. Lett. 25 (2012), 990-994.

[15] Q.-H. Xu, H.-G. Xiao and H. M. Srivastava, A certain general subclass of analytic and bi-univalent functions and associated coefficient estimate problems, Appl. Math. Comput. 218 (2012), $11461-11465$

[16] Zhigang Peng and Qiuqiu Han, On the Coefficients of several classes of bi-uivalent functions, Acta. Math. Sci. 34B(1) (2014), 228-240.

[17] H. Tang, G-T. Deng and S-H. Li, Coeficient estimates for new subclasses of Ma-Minda bi-univalent functions, J. Inequal. Appl. 2013, 2013:317, 1-10 